\title{
Novel feasibilities for preparation of serum albumin-based core-shell nanoparticles in flow conditions
}

\author{
Alexandra N. Kovács ${ }^{1}$. Norbert Varga ${ }^{1}$ - Gyöngyi Gombár ${ }^{1}$ • Viktória Hornok ${ }^{1,2}$ • Edit Csapó ${ }^{1,3}$
}

Received: 22 January 2020 / Accepted: 27 February 2020 / Published online: 23 March 2020

(C) Akadémiai Kiadó 2020

\begin{abstract}
We first demonstrate a simple and rapid fabrication protocol of bovine serum albumin (BSA) nanoparticles (NPs), as potential drug carriers using a microchannel flow technique with the successful encapsulation of a water-soluble kynurenic acid (KYNA) having neuroactive property has also been performed. By comparison, the preparation of a hydrophobic $\alpha$-Tocopherol (TP)-loaded polylactide-co-glycolide 50:50 (PLGA50)-based NPs was also carried out under flow conditions. We highlight several benefits of the flow technique over the commonly known self-assembly and nanoprecipitation processes. The average particle diameter, the size distribution, the encapsulation efficiency (EE\%) and the drug release kinetics of these different core-shell type NPs prepared by the flow as well as the above-mentioned classic methods were compared. The decisive role of the flow rate (FR), the relative flow rate (RFR) of the components in the particle size of both BSA- and PLGA50-based NPs have also been verified. By utilization of the optimal flow conditions, the average size can be decreased with ca. 15-20\% and lower polydispersity index (PDI) can be also achieved. It was confirmed that the systematic change of the RFR values resulting in the controlled size of the drug-loaded BSA NPs between 120 and $140 \mathrm{~nm}$, while $\mathrm{d}=149 \mathrm{~nm}$ was obtained for self-assembled NPs. However, for BSA-based NPs quite similar EE\% was obtained for both methods (ca. 11-12\%), but for PLGA50/TP NPs the application of flow device increased the EE\% from $67.0 \%$ to $71.5 \%$.
\end{abstract}

Keywords Serum albumin $\cdot$ PLGA $\cdot$ Kynurenic acid $\cdot$ Tocopherol $\cdot$ Core-shell nanocarriers $\cdot$ Microfluidic device

\section{Introduction}

Several fabrication protocols are known in the literature for producing serum protein and polymeric (e.g. PLA, PLGA, chitosan, alginate etc.) nanoparticles (NPs) as potential colloidal drug delivery systems (DDs) in the modern pharmaceutical researches. In case of serum albumin NPs, the commonly used preparation protocol is the desolvation process [1], where

\footnotetext{
Highlights

- Controllable preparation of serum albumin nanocarriers containing kynurenic acid has been presented under flow conditions

- By optimized flow conditions, a reproducible and large-scale production of BSA-based core-shell nanoparticles can be obtained

- Utilization of flow device results in smaller particle sizes compared to self-assembly and nanoprecipitation methods
}

Electronic supplementary material The online version of this article (https://doi.org/10.1007/s41981-020-00088-4) contains supplementary material, which is available to authorized users.

Viktória Hornok

vhornok@chem.u-szeged.hu

Edit Csapó

juhaszne.csapo.edit@med.u-szeged.hu

1 Department of Physical Chemistry and Materials Science, Interdisciplinary Excellence Centre, University of Szeged, H-6720 Rerrich B. Square 1, Szeged, Hungary
2 MTA Premium Post Doctorate Research Program, University of Szeged, H-6720 Rerrich B. Square 1, Szeged, Hungary

3 Department of Medical Chemistry, MTA-SZTE Biomimetic Systems Research Group, Faculty of Medicine, University of Szeged, H-6720 Dóm Square 8, Szeged, Hungary 
a continuous dropwise addition of ethanol to an aqueous solution of albumin is carried out under continuous stirring in the absence or mainly in the presence of crosslinking agents like glutaraldehyde [2], carbodiimides [3] or others [4], etc. By utilizing crosslinkers, the aggregation of protein particles can be eliminated and depending on the structure and the solubility of the encapsulated drug(s), serum albumin-based NPs in the average diameter of ca. $80-1000 \mathrm{~nm}$ can be fabricated. The main disadvantage of this technique is the demand for the strict purification step(s), where the separation of different serum albumin fractions is necessary, and the removal of unreacted crosslinkers is also required. Beside the desolvation technique, the emulsification (using nano- [5] or mainly microemulsions [6]) is also widely used, which results in NPs with average diameter of $\sim 100-1000 \mathrm{~nm}$, but the thermal stabilization [7] of the albumin nanospheres or the application of stabilizing agents like surfactants [8] or crosslinking molecules (e.g. 2,3-butadiene or formaldehyde [8]) are indispensable for this technique. This process requires organic solvents and the removal of both the surfactants and oily residues is problematic. Moreover, the thermal gelation [9], the nanospray drying [10], the gamma irradiation [11], the albuminbound technology (nab-technology) [12] or the self-assembly [13] techniques are also known for fabrication of different drugcontaining albumin NPs. Comparing the mentioned techniques, we can conclude that the thermal stabilization is only suitable for encapsulating drug molecules that are not heat sensitive. However, the nano spray drying is a simple and scalable technique, but it produces larger particles in the size range of $0.5-3 \mu \mathrm{m}$ and high pressure is necessary for preparation of albumin NPs using nabtechnology. For the preparation of polymeric NPs, the emulsification [1] the thermal gelation [14], the self-assembly [1] as well as the desolvation or nanoprecipitation [15-17] techniques are also commonly applied; the benefits and difficulties of these methods are summarized above. Beside the mentioned well-known protocols, the microfluidic technology provides an alternative strategy for the fabrication of both polymeric and serum albumin NPs. Reviewing the articles in the literature, it can be stated that, while for polymers, several publications summarize the benefits of the flow process like tunable size, structure and surface, narrow size distribution, precisely controlled release profile as well as the possibility of the encapsulation of water-soluble and insoluble drugs [18-21], but no articles are found relating to the fabrication of core-shell type, drug-loaded albumin-based NPs prepared by microfluidic technique.

In this actual work, we firstly demonstrate the preparation possibility of BSA protein-based NPs in a simple flow system, where the encapsulation of a hydrophilic kynurenic acid (KYNA) having neuroactive feature $[22,23]$ has been also carried out. Our previous published work [24], which clearly summarizes the preparation of a promising nanosized BSAbased drug delivery product containing KYNA - fabricated by self-assembly technique - is a patented invention (Hungarian patent, P1500356) [25]. This reason also strongly confirms the demand of the development of a new, controllable preparation process, that results in particles with tunable size and narrow size distribution. The microfluidic strategy may offer an excellent opportunity for automatized large-scale pharmaceutical production. Moreover, the preparation of PLGA50 polymer-based NPs has also been carried out under flow conditions, where the polymeric NPs were loaded with a hydrophobic tocopherol (TP) molecules and the results of the hydrophilic albumin- and the hydrophobic polymeric systems were compared and interpreted. The present investigation also aimed to compare the results obtained from this simple flowbased and the classic preparation protocols like self-assembly and desolvation or nanoprecipitation, therefore we explored the effect of the preparation parameters (flow rate, relative flow rate, pressure) on the average particle diameter, size distribution, drug loading (DL\%), EE\% and drug release kinetics of the different drug-nanocarrier systems.

\section{Experimental}

\section{Materials}

Bovine serum albumin (BSA, $\sim 66.000$ Da molecular weight), kynurenic acid (KYNA, $\geq 98 \%),( \pm)-\alpha$-Tocopherol (TP, $\geq$ 96\%), Pluronic-F127 (PLUR, $\geq 99 \%$ ), poly(allylamine hydrochloride (PAH, $\mathrm{M}_{\mathrm{w}} \sim 15.000 \mathrm{Da}$ ), poly(lactide-co-glycolide acid) copolymer (PLGA50, $\geq 99 \%$ at 50:50 monomer ratio, $\mathrm{Mw}=30.000-60.000 \mathrm{Da})$ and sodium dihydrogen phosphate monohydrate $\left(\mathrm{NaH}_{2} \mathrm{PO}_{4} \times \mathrm{H}_{2} \mathrm{O}, \geq 99 \%\right)$ were purchased from Sigma-Aldrich. Disodium hydrogen phosphate $\left(\mathrm{Na}_{2} \mathrm{HPO}_{4}\right.$, $\geq 99 \%$ ), sodium chloride $(\mathrm{NaCl}, \geq 99 \%)$ and sodium hydroxide pastilles $(\mathrm{NaOH}, \geq 96 \%)$ were purchased from Molar Chemicals Ltd. Highly purified water (MQ) was used during the experiments obtained by deionization and filtration with a Millipore purification apparatus $\left(18.2 \mathrm{M} \Omega \cdot \mathrm{cm}\right.$ at $\left.25^{\circ} \mathrm{C}\right)$. All reagents and solvents used were of analytical grade.

\section{Preparation of BSA-based NPs containing KYNA and TP}

Throughout the bulk syntheses of core-shell NPs, all the samples were prepared in phosphate buffer saline (PBS, $\mathrm{pH}=$ 7.40) solution at $25{ }^{\circ} \mathrm{C}$ according to the procedure published earlier [24] with a minor modification. Firstly, $24.0 \mathrm{mg}$ of BSA was dissolved in $8.0 \mathrm{~mL}$ PBS and stirred (350 rpm) until it completely dissolved. Then, $13.82 \mathrm{mg}$ of KYNA was added to this solution; after $2 \mathrm{~h}$ of stirring the $2 \mathrm{~mL}$ of aqueous solution of the polyelectrolyte PAH $(\mathrm{c}=2.01 \mathrm{mg} / \mathrm{mL})$ was administered to the reaction mixture (drop by drop). The same preparation method and amounts were used for the encapsulation of TP in BSA/PAH complex. 


\section{Preparation of PLGA50-based NPs containing KYNA and TP}

The PLGA/TP and PLGA/KYNA NPs were prepared by nanoprecipitation method [16]. 5.0 mg of PLGA50 was dissolved in $1.0 \mathrm{~mL}$ of acetone. This solution was added dropwise $(10 \mu \mathrm{L} / 5 \mathrm{~s})$ into the $10 \mathrm{~mL}$ of aqueous solution of PLUR stabilizer $(\mathrm{c}=0.1 \mathrm{mg} / \mathrm{mL})$ under $1000 \mathrm{rpm}$ magnetic stirring at room temperature. In case of the preparation of the drug-loaded NPs, $1.25 \mathrm{mg}$ of TP was dissolved in the acetone phase, while the KYNA $(1.25 \mathrm{mg})$ was dispersed in the aqueous phase, and the dispersions were further stirred (350 rpm) for 2 days to remove the acetone by evaporation.

\section{Preparation of BSA-and PLGA50 core-shell NPs in microfluidic device}

The microfluidic syntheses were carried out in Syrris Asia Flow system (Syrris Ltd.) equipped with two syringe pumps and a direct connection to the $\mu$-mixer cell with a volume of $250 \mu \mathrm{L}$, and a pressure controller apparatus. After passing through the syringe pumps, the two solutions mixing in the $\mu$-mixer cell and then the sample collected in defined time intervals. The schematic representation of the preparation of the core-shell NPs by this microfluid device is presented in Scheme 1 . The FR and the RFR were adjusted easily during the synthesis of the drug-loaded NPs.

In case of BSA-containing core-shell NPs the same concentrations were used than for the self-assembly method. After the $2 \mathrm{~h}$ of stirring of the BSA/KYNA solution, the PAH containing solution was added to the channel 1 (v1), while the BSA- and drug-containing solution was filled into the channel 2 (v2). For the determination of the optimal RFR, in the first measurements the FR was kept constant $\left(\mathrm{v}_{2}=500 \mu \mathrm{L} / \mathrm{min}\right)$ while the aqueous PAH solution was varied in defined flow parameter intervals $\left(\mathrm{v}_{1}=25-150 \mu \mathrm{L} / \mathrm{min}\right)$. In a second experiment series the $\mathrm{v}_{1}: \mathrm{v}_{2}$ ratio of the solutions was kept constant $\left(\mathrm{v}_{1}: \mathrm{v}_{2}=1: 4\right)$, while the $\mathrm{v}_{1}$ and $\mathrm{v}_{2}$ values were continually increased. For both preparation methods, the NPs were separated by centrifugation at $5000 \mathrm{rpm}$ for $10 \mathrm{~min}$ and the samples were freeze-dried (Christ Alpha 1-2 LD plus) for $24 \mathrm{~h}$ and stored at $-20^{\circ} \mathrm{C}$.

For PLGA-containing core-shell NPs the organic phase (PLGA and TP in acetone) flowed in channel $1\left(\mathrm{v}_{1}\right)$, and in the other channel, the aqueous solution of PLUR is steamed $\left(\mathrm{v}_{2}\right)$. Firstly, the FR of the organic phase was kept constant $\left(\mathrm{v}_{1}\right.$; $25 \mu \mathrm{L} / \mathrm{min}$ ), while the aqueous phase was changed in the range of $\mathrm{v}_{2}=250-1250 \mu \mathrm{L} / \mathrm{min}$. In the next step, the $\mathrm{v}_{1}: \mathrm{v}_{2}$ ratio of the solutions was fixed $\left(\mathrm{v}_{1}: \mathrm{v}_{2}=1: 10\right)$ and the FR values were increased. During the washing step, the prepared samples were centrifuged at $12000 \mathrm{rpm}\left(15 \mathrm{~min}, 25^{\circ} \mathrm{C}\right)$ and the supernatant was removed; this step was repeated three times, then the purified NPs were lyophilized.

\section{Characterization of the prepared core-shell NPs}

Dynamic light scattering (DLS) studies were performed in HORIBA SZ-100 NanoParticle Analyzer (Retsch Technology GmbH, Germany) at room temperature. The light source was a diode pumped frequency doubled laser $(\lambda=$ $532 \mathrm{~nm}, 10 \mathrm{~mW}$ ) and photomultiplier tubes (PMT) were used as the detector at $90^{\circ}$ scattering angle. For the measurements, the viscosity $(\eta, \mathrm{mPa} \cdot \mathrm{s}$ or $T(\mathrm{~K})$ dependent equation) of the solvent and the refractive index of the measured particles must be given, then the photon correlation method is used to acquire the auto correlation function $G 2(\tau)$ vs. scattering light intensity $I(\mathrm{t})$. To obtain the particle size distribution, the software uses the cumulant method for calculation, then the

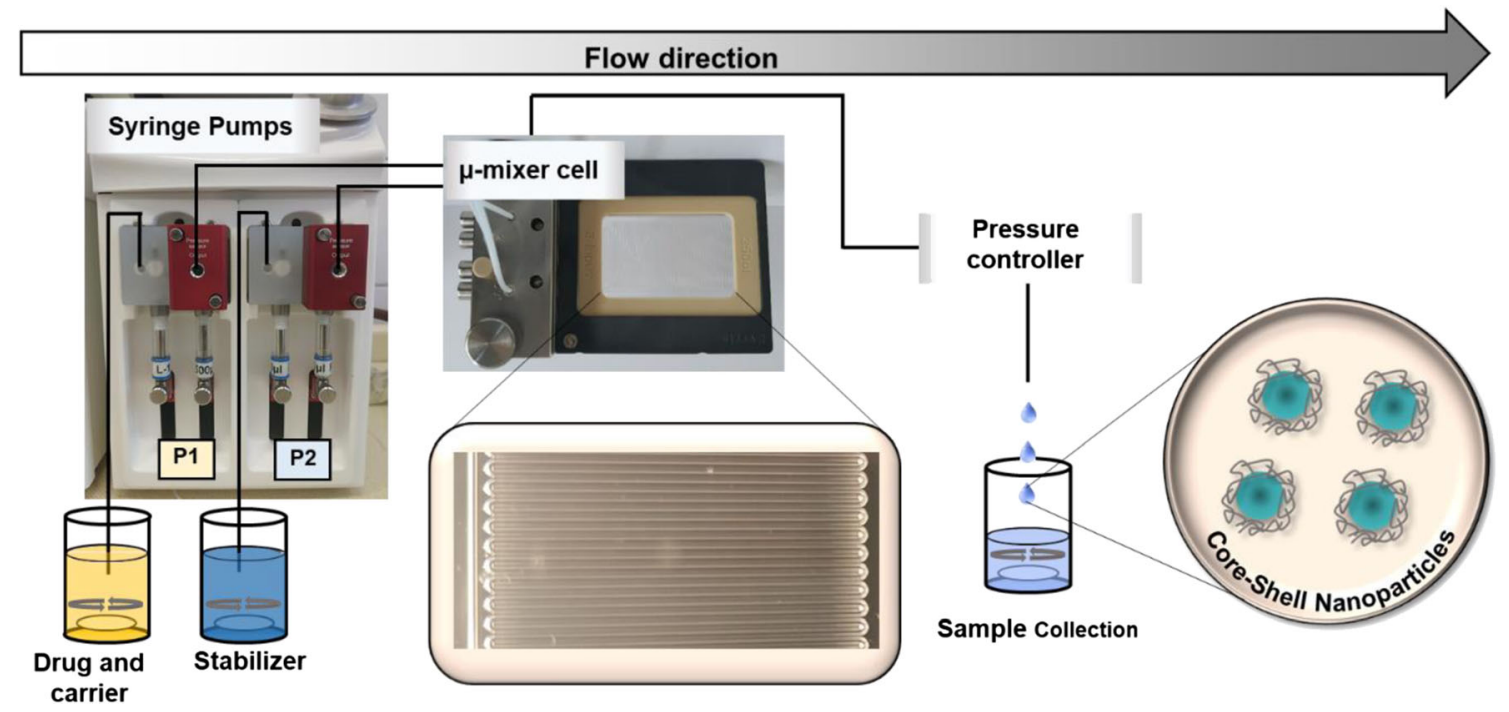

Scheme 1 Schematic representation of the used flow system with the pumps and the $\mu$-mixer cell 
histogram method is applied to get the mean and the standard deviation of the distribution function from the average of 10 separate measurement form each sample.

The transmission electron microscopy (TEM) images were registered by using a Jeol JEM-1400plus equipment (Japan) at $120 \mathrm{keV}$ accelerating voltage. For the determination of the DL\% and the EE\% the absorbance spectra of the encapsulated drugs (KYNA and TP molecules) were registered by spectrophotometric measurements in a range of $200 \mathrm{~nm}$ to $600 \mathrm{~nm}$ with the use of a UV-1800 (Shimadzu) double beam spectrophotometer with a $1 \mathrm{~cm}$ quartz cuvette. The concentration of the encapsulated drug (the amount of KYNA and TP) was determined by using the classical calibration curves (Fig. S1S2) [16]. The characteristic absorbance band for the TP is $\lambda=$ $294 \mathrm{~nm}$, while for KYNA is $\lambda=332 \mathrm{~nm}$. The well-known DL\% ad EE\% values were calculated by Eqs. 1 and 2.

$$
\begin{aligned}
& \text { DL\% }=\frac{\text { encapsulated mass of drug }}{\text { total mass of the nanoparticles }} \\
& \text { EE\% }=\frac{\text { encapsulated mass of drug }}{\text { total mass of the in synthesis }}
\end{aligned}
$$

\section{In vitro release studies}

The in vitro drug release experiments were carried out in PBS $(\mathrm{pH}=7.4,0.9 \mathrm{w} / \mathrm{w} \% \mathrm{NaCl})$ at $37{ }^{\circ} \mathrm{C}$ using a semipermeable cellulose membrane (Sigma-Aldrich). For the PLGA/TP system the poor solubility of TP was enhanced by the presence of PLUR in the release medium ( $c=1 \mathrm{mg} / \mathrm{mL}$ ) according to the studies published previously [16]. The release process was followed for $6 \mathrm{~h}$. Samples were taken every $10 \mathrm{~min}$ in the first hour and then $20 \mathrm{~min}$ and finally once per hour. The concentration of the KYNA and TP molecules in the release medium was determined by using calibration curves (Fig. S1, S3). Analysis of in vitro drug release data helps evaluate the release kinetics and the proposed mechanism. Numerous mathematical models (first-order, Weibull, Korsmeyer-Peppas, etc.) have been used to describe the release properties of the drug molecules $[16,26-28]$. The mentioned equations were summarized in Suppl. Inf.

\section{Results and discussion}

\section{Characterization of the KYNA- and TP-containing BSA- and PLGA50 nanocarriers prepared by self-assembly and nanoprecipitation}

To compare the different preparation protocols, firstly, the BSA- and PLGA50-based NPs have been prepared by the classical methods like self-assembly and nanoprecipitation. The average diameters of the unloaded, the KYNA- and TP-loaded NPs prepared by the classical wet chemical techniques were determined by DLS for both carrier systems; the representative DLS curves are presented in Fig. 1. The average diameter of the drugfree PLGA50 NPs is $d=124 \pm 33 \mathrm{~nm}$, while for polymeric NPs containing TP and KYNA the size increased to $d=$ $157 \pm 55 \mathrm{~nm}$ and $\mathrm{d}=198 \pm 50 \mathrm{~nm}$, respectively. However, no articles were found for PLG50/KYNA system, but a few publications reported PLGA50/TP NPs ranging from ca. $85 \mathrm{~nm}$ to $280 \mathrm{~nm}$, where the size strongly depends on the concentration and molecular weight of the applied polymer as well as the absence and the presence of surfactant stabilizer (e.g. no surfactant [29], sorbitan monooleate (Span80) [30], sodium dodecyl sulfate (SDS) or polyvinyl alcohol (PVA) [31]). The size of our PLGA50/TP NPs $(\mathrm{d}=157 \pm 55 \mathrm{~nm})$ stabilized by PLUR is in good agreement with this average diameter range. For albumin-containing drug delivery systems, the drug-free BSA/PAH complex dispose of the smallest size $(d=107$ $\pm 34 \mathrm{~nm}$ ), but the average diameter of the BSA/KYNA/ PAH NPs is $149 \pm 44 \mathrm{~nm}$. In case of the capsulation of TP the size is increased to ca. $300 \mathrm{~nm}$. Comparing the average size of the NPs as well as the solubility/ hydrophobicity of the carriers and the encapsulated molecules, it can be stated that the serum proteinpolyelectrolyte complex is the optimal carrier for the neuroactive KYNA, which was also published previously [24], while the PLGA50-PLUR polymer-surfactant forms smaller nanocarriers containing TP molecules having hydrophobic nature.

To get comprehensive knowledge about the attribution of the delivery system, both the structure and the shape of the composites were investigated by TEM, the representative images are shown in Fig. 2.

The core-shell like structures are easily recognizable in the optimized PLGA50/TP and BSA/KYNA/PAH composites, suggesting the successful encapsulation of the therapeutic agents. The average diameters of these NPs determined by TEM images are in good correspondence with the results of DLS studies; for pure PLGA50 $=117 \pm 30 \mathrm{~nm}$ is obtained, while for TP-containing PLGA50 NPs $d=148 \pm 35 \mathrm{~nm}$ is measured. For BSA-based NPs, $\mathrm{d}=102 \pm 40 \mathrm{~nm}$ and $158 \pm$ $40 \mathrm{~nm}$ values were calculated before and after loading with KYNA. In agreement with the obtained DLS results of the PLGA50/KYNA and BSA/TP/PAH systems, the efficiency of the drug capsulation into the carriers is low and the formation of core-shell like structures are not recognizable based on TEM images (Fig. S4). Based on these results, in case of the preparation of these systems by using microfluidic device only the PLGA50/TP and BSA/KYNA/PAH composites are studied in detail. 
Fig. 1 Particle size distribution curves of the polymer-(a), and the protein-based NPs (b) with and without drug molecules

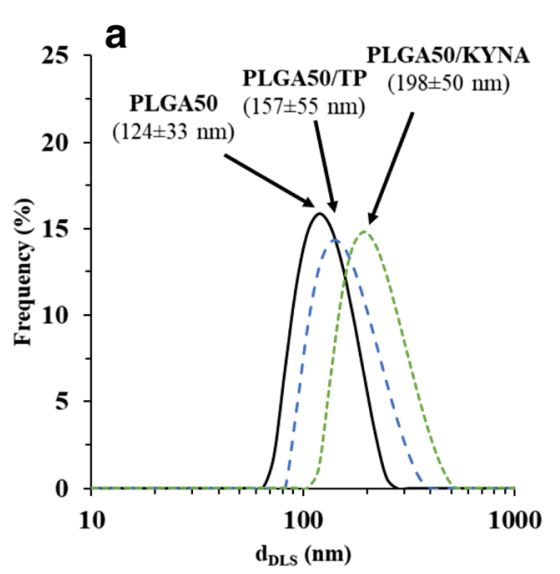

\section{Drug encapsulation in BSA/PAH and PLGA50/PLUR NPs in flow system}

Based on the experimental results of the previous chapter, the fabrication possibilities of the BSA/KYNA/PAH and the PLGA50/TP systems have been carried out by using a microfluidic device. In the literature there is no reference for the fabrication of serum-albumin NPs under flow conditions, therefore the successful production of BSA-based core-shell NPs was the main task of this work. However, several articles can be found for the fabrication of PLGA50-based drug delivery systems using microfluidic process, but no paper is available to implement the TP encapsulation in a flow system. In the first step, in order to find the optimal parameters for the flow-based preparation the flow parameters (FR and RFR) of the different channels $\left(\mathrm{v}_{1}\right.$ and $\left.\mathrm{v}_{2}\right)$ were varied. In case of the PLGA50/TP system the appropriate FR and RFR values together with the average size of the formed NPs as well as the polydispersity index (PI) determined by DLS are summarized in Table 1.

The increase of the FR of the aqueous phase (PLUR containing) from $250 \mu \mathrm{L} / \mathrm{min}$ to $1250 \mu \mathrm{L} / \mathrm{min}$ results in the slight increase in the size of the TP-free sample (from $144 \mathrm{~nm}$ to $167 \mathrm{~nm}$ ). For TP-containing PLGA50 NPs only a few nm changes were observed (148 $\mathrm{nm}$ to $155 \mathrm{~nm}$ ). Using constant RFR (1:10), but different FR (see Table 1) the average size varies in the range of ca. 130-160 $\mathrm{nm}$ for both samples. The optimal flow parameter, where the size is the smallest $(\mathrm{d}=$ $134 \mathrm{~nm})$, is $75: 750(\mu \mathrm{L} / \mathrm{min})$. The TEM measurements unambiguously confirm this observation, because the formation of core-shell structure is clearly detectable using $75: 750 \mu \mathrm{L} / \mathrm{min}$ (Fig. 3b, $d=132 \pm 22 \mathrm{~nm}$ ). The TEM images of the PLGA50/ TP NPs prepared at different FR are shown in Fig. 3.

At low FR (Fig. 3a, 25:250 $\mu \mathrm{L} / \mathrm{min}$ ) the formation of the core-shell structure is not observed, but at high flow
Fig. 2 Characteristic TEM images of the polymer- and protein-based NPs with and without drug molecules
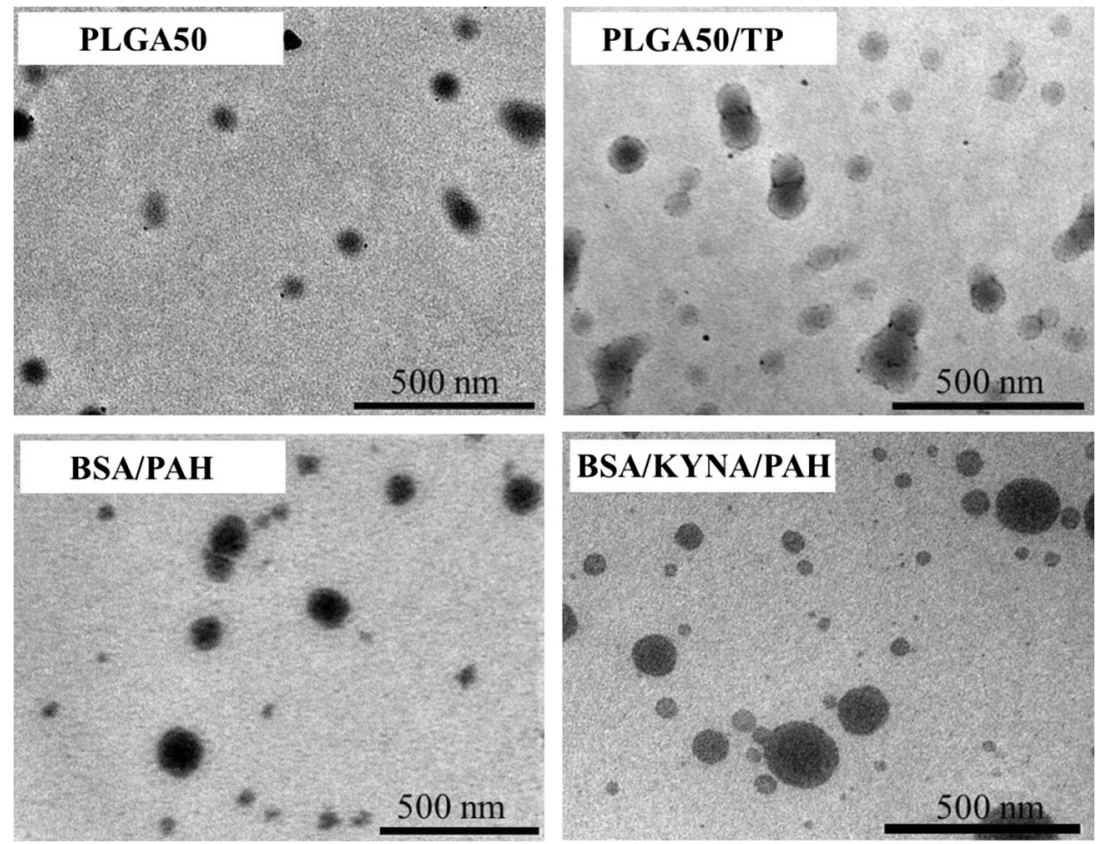
Table 1 Average particle diameter $\left(d_{D L S}\right)$ and polydispersity index $(P I)$ of PLGA-based samples at different flow conditions $\left(v_{1}, v_{2}\right)$ determined from DLS measurements

\begin{tabular}{|c|c|c|c|c|c|c|}
\hline \multirow[t]{2}{*}{ PLGA50 (TP) } & \multirow[t]{2}{*}{ PLUR } & \multirow[t]{2}{*}{ RFR } & \multicolumn{2}{|l|}{ 'TP-free } & \multicolumn{2}{|l|}{ TP-loaded } \\
\hline & & & PLGA50 & & PLGA50 & \\
\hline $\mathrm{v}_{1}(\mu \mathrm{L} / \mathrm{min})$ & $\mathrm{v}_{2}(\mu \mathrm{L} / \mathrm{min})$ & $\mathrm{v}_{1}: \mathrm{v}_{2}$ & $\mathrm{~d}_{\mathrm{DLS}}^{\mathrm{a}}(\mathrm{nm})$ & PI & $\mathrm{d}_{\mathrm{DLS}}{ }^{\mathrm{a}}(\mathrm{nm})$ & PI \\
\hline 25 & 250 & $1: 10$ & $144 \pm 37$ & $0.07 \pm 0.03$ & $148 \pm 31$ & $0.07 \pm 0.03$ \\
\hline 25 & 500 & $1: 20$ & $147 \pm 35$ & $0.09 \pm 0.04$ & $144 \pm 30$ & $0.05 \pm 0.03$ \\
\hline 25 & 750 & $1: 30$ & $153 \pm 31$ & $0.08 \pm 0.06$ & $153 \pm 30$ & $0.04 \pm 0.02$ \\
\hline 25 & 1000 & $1: 40$ & $177 \pm 51$ & $0.13 \pm 0.03$ & $153 \pm 38$ & $0.04 \pm 0.03$ \\
\hline 25 & 1250 & $1: 50$ & $167 \pm 57$ & $0.17 \pm 0.06$ & $155 \pm 33$ & $0.04 \pm 0.02$ \\
\hline 25 & 250 & $1: 10$ & $144 \pm 37$ & $0.07 \pm 0.03$ & $148 \pm 31$ & $0.07 \pm 0.03$ \\
\hline 50 & 500 & $1: 10$ & $137 \pm 36$ & $0.10 \pm 0.06$ & $141 \pm 32$ & $0.11 \pm 0.03$ \\
\hline 75 & 750 & $1: 10$ & $142 \pm 35$ & $0.09 \pm 0.04$ & $134 \pm 27$ & $0.09 \pm 0.0$ \\
\hline 100 & 1000 & $1: 10$ & $132 \pm 33$ & $0.10 \pm 0.03$ & $167 \pm 48$ & $0.15 \pm 0.02$ \\
\hline 150 & 1500 & $1: 10$ & $159 \pm 44$ & $0.10 \pm 0.06$ & $157 \pm 43$ & $0.10 \pm 0.03$ \\
\hline
\end{tabular}

${ }^{\mathrm{a}}$ the experimental error of the peak maximum is below $2.5 \%$ parameters (Fig. 3c, 150:1500 $\mu \mathrm{l} / \mathrm{min}$ ) the average size is increased $(\mathrm{d}=163 \pm 14 \mathrm{~nm})$ and the formation of only a thin shell is observed around the higher core of the particles. For the BSA/KYNA/PAH NPs no significant change could be observed in the particle morphology at different FR and RFR, but the size varied in the range of ca. 120-185 nm. The effect of RFR in the BSA-containing carriers was also investigated, the results are shown in Table 2. If we compare the NPs prepared by the two technique, some morphological differences can be observed. In case of the BSA-based nanocomposites prepared by the microfluidic technique, a more distinct core-shell structure is observed and the polyelectrolyte shell is thicker. The KYNA-free NPs are ca. 20\% smaller than the KYNA-loaded BSA/PAH complexes. For the BSA/ KYNA/PAH NPs, the smallest average size is obtained at 125:500 $\mu \mathrm{L} / \mathrm{min}$ ratio (Fig. $3 \mathrm{~d}, \mathrm{v}_{1}: \mathrm{v}_{2}=1: 4, \mathrm{~d}=121 \pm 42 \mathrm{~nm}$.
The effect of FR is investigated in the different channels using this 1:4 ratio. Under this condition, the BSA/PAH composite is very sensitive to the FR, from the use of 25:100 to $150: 600 \mu \mathrm{L} / \mathrm{min}$ the $\mathrm{d}_{\text {DLS }}$ systematically and significantly decreased from $147 \pm 51 \mathrm{~nm}$ to $90 \pm 24 \mathrm{~nm}$ in the case of KYNA-free NPs, while from $183 \pm 56$ (Fig. 3e) to $129 \pm$ $49 \mathrm{~nm}$ for KYNA-loaded samples, respectively.

In accordance with the DLS results of the batch- and the flow-based fabrication processes, a significant difference in the particle size is noticed. Compared to the flow method prepared particles $(121 \pm 42 \mathrm{~nm}$ for BSA/KYNA/PAH and $134 \pm 27 \mathrm{~nm}$ for PLGA/TP) to the bulk phase reaction (158 $\pm 40 \mathrm{~nm}$ for BSA/KYNA/PAH, $148 \pm 35 \mathrm{~nm}$ for PLGA/TP) it can be stated that the flow-based preparation method results about ca. 24 and $10 \%$ smaller particle size than the bulk phase process, while the PI does not change significantly. Significant

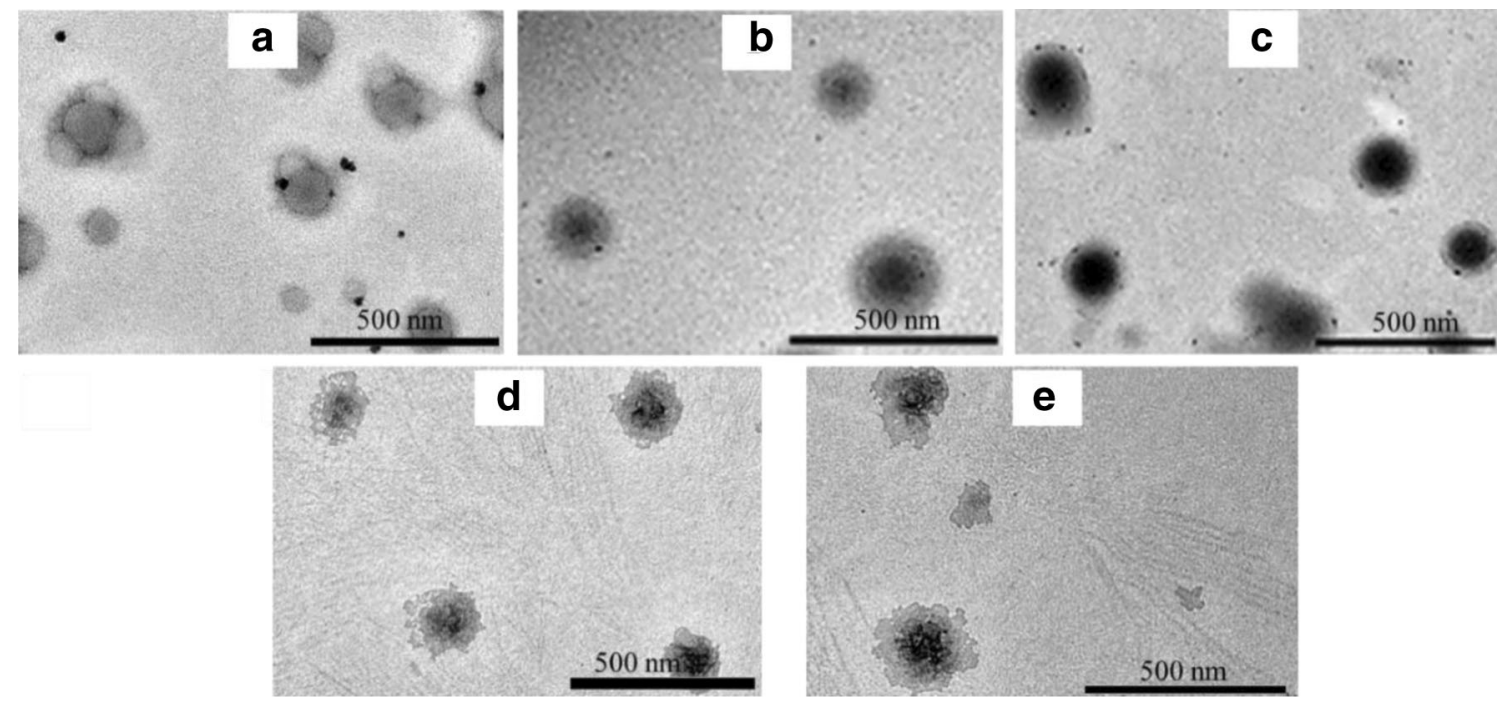

Fig. 3 Characteristic TEM images of the prepared NPs using different $\mathrm{v}_{1}: \mathrm{v}_{2}$ flow rates. PLGA/TP NPs: a 25:250, b 75:750, c 150:1500; BSA/KYNA/ PAH NPs: d 125:500, e 25:100 
Table 2 Average particle diameter $\left(d_{D L S}\right)$ and polydispersity index $(P I)$ of BSAbased samples at different flow $\left(v_{1}, v_{2}\right)$ conditions determined from DLS measurements

\begin{tabular}{|c|c|c|c|c|c|c|}
\hline \multirow[t]{2}{*}{ PAH } & \multirow[t]{2}{*}{ BSA (KYNA) } & \multirow[t]{2}{*}{ RFR } & \multicolumn{2}{|l|}{ KYNA-free } & \multicolumn{2}{|c|}{ KYNA-loaded } \\
\hline & & & \multicolumn{2}{|l|}{ BSA/PAH } & \multicolumn{2}{|l|}{ BSA/PAH } \\
\hline $\mathrm{v}_{1}(\mu \mathrm{L} / \min )$ & $\mathrm{v}_{2}(\mu \mathrm{L} / \min )$ & $\mathrm{v}_{1}: \mathrm{v}_{2}$ & $\mathrm{~d}_{\mathrm{DLS}}^{\mathrm{a}}(\mathrm{nm})$ & PI & $\mathrm{d}_{\mathrm{DLS}}^{\mathrm{a}}(\mathrm{nm})$ & PI \\
\hline 25 & 500 & $1: 20$ & $114 \pm 44$ & $0.31 \pm 0.03$ & $142 \pm 66$ & $0.26 \pm 0.04$ \\
\hline 50 & 500 & $1: 10$ & $93 \pm 30$ & $0.18 \pm 0.06$ & $137 \pm 45$ & $0.22 \pm 0.05$ \\
\hline 75 & 500 & $1: 6.7$ & $100 \pm 28$ & $0.20 \pm 0.03$ & $139 \pm 51$ & $0.19 \pm 0.03$ \\
\hline 100 & 500 & $1: 5$ & $116 \pm 37$ & $0.18 \pm 0.10$ & $122 \pm 36$ & $0.25 \pm 0.03$ \\
\hline 125 & 500 & $1: 4$ & $103 \pm 28$ & $0.20 \pm 0.04$ & $121 \pm 42$ & $0.27 \pm 0.04$ \\
\hline 150 & 500 & $1: 3.3$ & $117 \pm 38$ & $0.21 \pm 0.02$ & $123 \pm 42$ & $0.28 \pm 0.04$ \\
\hline 25 & 100 & $1: 4$ & $147 \pm 51$ & $0.17 \pm 0.03$ & $183 \pm 56$ & $0.27 \pm 0.02$ \\
\hline 50 & 200 & $1: 4$ & $150 \pm 48$ & $0.16 \pm 0.03$ & $176 \pm 62$ & $0.27 \pm 0.06$ \\
\hline 75 & 300 & $1: 4$ & $139 \pm 44$ & $0.15 \pm 0.03$ & $158 \pm 57$ & $0.28 \pm 0.07$ \\
\hline 100 & 400 & $1: 4$ & $130 \pm 38$ & $0.19 \pm 0.03$ & $154 \pm 49$ & $0.29 \pm 0.04$ \\
\hline 125 & 500 & $1: 4$ & $103 \pm 28$ & $0.20 \pm 0.04$ & $121 \pm 42$ & $0.27 \pm 0.04$ \\
\hline 150 & 600 & $1: 4$ & $90 \pm 24$ & $0.18 \pm 0.04$ & $129 \pm 49$ & $0.33 \pm 0.06$ \\
\hline
\end{tabular}

${ }^{\mathrm{a}}$ the experimental error of the peak maximum is below $2.5 \%$ changes in the PI are not obtained for BSA/KYNA/PAH (PI = 0.269 and 0.226 for the low- and batch-based samples, respectively), but in case of PLGA50/TP NPs the PI values decrease to ca. 0.1. It can be confirmed that the preparation of serum albumin NPs containing a neuroactive KYNA was successful and by optimized concentrations as well as flow conditions a reproducible and large-scale production of these NPs was achieved. Besides the size, morphology and polydispersity of the nanocarrier particles, the DL\% and EE\% were also determined for the two drug carrier systems. However, in case of BSA/PAH NPs similar values, $12.1 \%$ and $11.7 \%$ of KYNA molecules were encapsulated using the batch- and flow preparation methods (5.4\% and $5.2 \%$ of DL\%), respectively, but significantly higher differences were achieved for the hydrophobic drug-polymer/surfactant carrier system. Namely, $67.1 \%$ and $71.5 \%$ EE\% (14.4\% and $15.2 \%$ DL\%) were obtained for PLGA50/TP NPs prepared by nanoprecipitation and under flow conditions, respectively. According to the above presented data the encapsulation was slightly more successful for PLGA50/TP system using optimized flow conditions. Comparing the encapsulation results with the data in the literature [29, 31], we can confirm that, our developed flow-based fabrication protocol has some benefits. The obtained $71.5 \% \mathrm{EE} \%$ is significantly higher than the $45 \%$ for the $85 \mathrm{~nm}$-sized PLGA50/TP NPs published by Murugeshu et al. [29]. Their relatively low value may be due to the lack of stabilizing molecules. Zigoneanu et al. published higher, 88 95\% EE\% for similar PLGA50/TP system depending on the applied SDS surfactant and PVA polymer, as stabilizing agents [31]. Effective drug carrier systems require the use of biocompatible materials for the fabrication, which is not satisfied by the SDS they use. We applied PLUR for stabilization of the drug-loaded polymeric NPs, which surfactant is commonly utilized in food production.

\section{Release studies}

After the characterization of the PLGA50/TP and BSA/ KYNA/PAH NPs, we aimed to determine the dissolution profiles of different drugs from the NPs. The registered dissolution curves are presented in Fig. 4.

When the flow technique is used for preparation of the albumin-based NPs, almost $80 \%$ of the encapsulated drug is released (Fig. 4A) from the BSA/KYNA/PAH NPs with a diameter of $\sim 120 \mathrm{~nm}$, while a slower drug release can be observed from the self-assembly-synthesized, $150 \mathrm{~nm}$-sized drug-loaded carriers; the maximum amount of the released KYNA is about $45 \%$ (Fig. 4B) within the examined 360 min time interval. Most probably the smaller size may facilitate the faster release of the encapsulated KYNA, which is a beneficial property of this composite. Previous in vitro as well as in vivo studies [24] clearly supported the successful penetration of

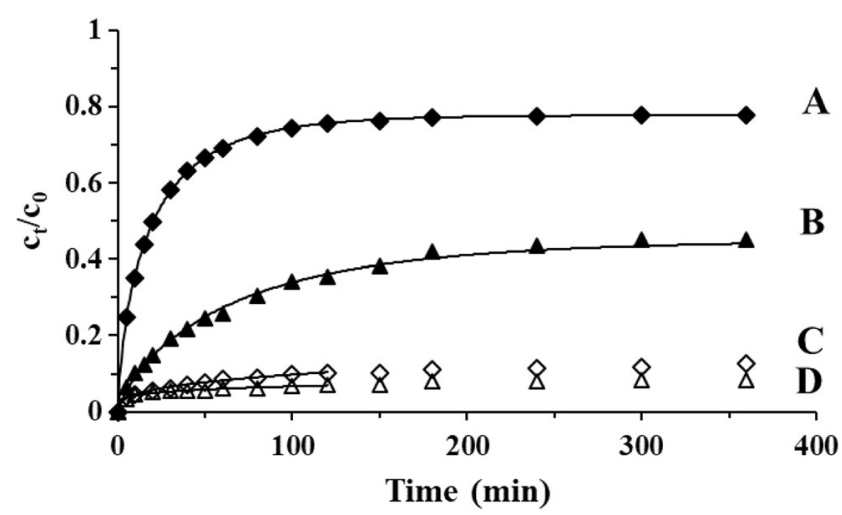

Fig. 4 Release profiles and different kinetic-models predicted (Weibull model (A, B) and Korsmeyer-Peppas model (C, D) release curves of KYNA (A: BSA/KYNA/PAH (flow); B: BSA/KYNA/PAH (selfassembly) and TP (C: PLGA50/TP (flow); D: PLGA50/TP (nanoprecipitation)) from the appropriate core-shell NPs $(\mathrm{pH}=7.4$, in PBS) 
Table 3 Parameters of the dissolution of TP and KYNA derived from the fitting of the release curves by several kinetic models

\begin{tabular}{|c|c|c|c|c|c|c|c|c|}
\hline \multirow{2}{*}{$\begin{array}{l}\text { Sample } \\
\text { Model }\end{array}$} & \multicolumn{2}{|l|}{ First-order } & \multicolumn{3}{|c|}{ Korsmeyer-Peppas } & \multicolumn{3}{|l|}{ Weibull } \\
\hline & $\mathrm{k}\left(\min ^{-1}\right)$ & $\mathrm{R}^{2}$ & $\mathrm{k}_{\mathrm{m}}\left(\min ^{-\mathrm{n}}\right)$ & $\mathrm{n}$ & $\mathrm{R}^{2}$ & $\mathrm{a}$ & $\mathrm{b}$ & $\mathrm{R}^{2}$ \\
\hline BSA/KYNA/PAH (flow) & 0.0229 & 0.9708 & 0.217 & 0.346 & 0.9843 & 8.201 & 0.707 & 0.9998 \\
\hline BSA/KYNA/PAH (self-assembly) & 0.0137 & 0.9884 & 0.068 & 0.527 & 0.9973 & 27.456 & 0.793 & 0.9954 \\
\hline PLGA50/TP (flow) & 0.0088 & 0.9497 & 0.168 & 0.333 & 0.9934 & 8.367 & 0.545 & 0.9882 \\
\hline PLGA50/TP (nanoprecipitation) & 0.0115 & 0.9189 & 0.320 & 0.197 & 0.9793 & 4.164 & 0.430 & 0.9728 \\
\hline
\end{tabular}

this albumin-based composites across the blood-brain barrier, where the fast release is effective in contrast to the controlled drug dissolution. In case of PLGA50/TP systems prepared by flow technique and nanoprecipitation, in the $360 \mathrm{~min}$ time period the drug availability in the release medium is $12 \%$ (Fig. 4C) and 8\% (Fig. 4D) for PLGA50/TP samples, respectively. These dissolution data are in good agreement with our previous observations [16]. Namely, for poly(lactic acid) (PLA), PLGA75 and PLGA65 carriers which have decreasing hydrophobicity in this order, $34 \%, 25 \%$ and $18 \%$ of the encapsulated TP can be released from the composites, respectively. By further reducing the hydrophobicity of the PLGA carrier, for PLGA50 the obtained $12 \%$ and $8 \%$ values are realistic. However, Zigoneanu et al. reported 88-95\% EE\% for the same PLGA50/TP NPs, but $35 \%$ of the encapsulated TP was released from their composite in the first hour. Naturally, the drug release profile depends on many parameters not only on the hydrophobicity but on the average particle diameter, polydispersity, density etc. $[32,33]$. To gain more information about our drugcarrier systems, we determined the release kinetics by investigating the applicability of various kinetic models like Weibull, Korsmeyer-Peppas and first-order models. The main parameters are summarized in Table 3.

The measured data of the drug release fit well to the Weibull expression, especially in case of the BSA-based system. For the PLGA system, no significant difference can be obtained in the release curves, but better results were detected when fitting the $60 \%$ of the primer data points with Korsmeyer-Peppas model. The drug release is diffusion controlled, the higher diffusion dissolution index (n) values (increase from 0.197 to 0.333 from nanoprecipitation to flow method) also confirms the more homogeneous size distribution of the particles, as obtained from the DLS. The value of $n$ is lower than 0.42 , that support the erosion of the polymercarrier is negligible.

\section{Conclusion}

The promising application of a simple flow-based preparation protocol for the production of colloidal drug-containing serum albumin protein and biocompatible PLGA50 polymer core-shell NPs has been demonstrated. To the best of our knowledge, this work is the first presenting the successful utilization of a flow preparation strategy for the fabrication of serum album nanocarriers with an average diameter of ca. $100 \mathrm{~nm}$. Our measurements emphasize the importance of the optimization of the flow-preparation method; well-chosen experimental parameters can be used to finetune the characteristic properties of the NPs like the product size and distribution, the DL\% and EE\% and the encapsulated drug sustainability. Our studies highlighted that the flow-based method results in NPs of ca. 15-20\% smaller particle size compared to the widely applied bulk-phase methods (e.g. self-assembly or nanoprecipitation) for both carriers. In case of the BSA/KYNA/PAH NPs prepared by the microfluidic device the encapsulated drug is released almost two times faster than from the bulk phase prepared samples, which makes the use of the microfluidic device more advantageous if rapid neuroactive drug dissolution is required. For the PLGA/TP system, the drug sustainability is not changed significantly by modification of the preparation methods (about $12 \%$ of the encapsulated amount released within $360 \mathrm{~min}$ ). We can conclude from the evaluation of the dissolution kinetics, that for the PLGA/TP NPs the most decisive process is the diffusion, while for the BSA/KYNA/PAH nanocarrier, besides the diffusion, the erosion of the carrier system also plays an important role in the release of encapsulated KYNA. Owing to the abovementioned features, after adjusting the parameters, the flowbased preparation method may offer an alternative opportunity for a reproducible, tunable and automatized large-scale pharmaceutical production.

Acknowledgements The research was supported by the National Research, Development and Innovation Office-NKFIH through the project GINOP-2.3.2-15-2016-00034 and FK 131446. V. Hornok acknowledges the Premium Post Doctorate Research Program of the Hungarian Academy of Sciences for the financial support. This paper was supported by the János Bolyai Research Scholarship of the Hungarian Academy of Sciences (E. Csapó). The Ministry of Human Capacities, Hungary grant TUDFO/47138-1/2019-ITM is acknowledged.

\section{Compliance with ethical standards}

Conflict of interest The authors declare no conflicts of interest. 


\section{References}

1. Nicolas J, Mura S, Brambilla D, MacKiewicz N, Couvreur P (2013) Design, functionalization strategies and biomedical applications of targeted biodegradable/biocompatible polymer-based nanocarriers for drug delivery. Chem Soc Rev 42:1147-1235

2. Merodio M, Arnedo A, Renedo MJ, Irache JM (2001) Ganciclovirloaded albumin nanoparticles: characterization and in vitro release properties. Eur J Pharm Sci 12:251-259

3. De Paoli Lacerda SH, Ingber B, Rosenzweig N (2005) Structurerelease rate correlation in collagen gels containing fluorescent drug analog. Biomaterials 26:7164-7172

4. Elzoghby AO, Samy WM, Elgindy NA (2012) Albumin-based nanoparticles as potential controlled release drug delivery systems. J Control Release 157:168-182

5. Donsì F, Senatore B, Huang Q, Ferrari G (2010) Development of novel pea protein-based nanoemulsions for delivery of nutraceuticals. J Agric Food Chem 58:10653-10660

6. Mehnert W, Mäder K (2001) Solid lipid nanoparticles: production, characterization and applications. Adv Drug Deliv Rev 47:165-196

7. Chunfu Z, Jinquan C, Duanzhi Y, Yongxian W, Yanlin F, Jiajü T (2004) Preparation and radiolabeling of human serum albumin (HSA)-coated magnetite nanoparticles for magnetically targeted therapy. Appl Radiat Isot 61:1255-1259

8. Pinto Reis C, Neufeld RJ, Ribeiro AJ, Veiga F (2006) Nanoencapsulation I. Methods for preparation of drug-loaded polymeric nanoparticles. Nanomed Nanotechnol Biol Med 2:8-21

9. Yu S, Yao P, Jiang M, Zhang G (2006) Nanogels prepared by selfassembly of oppositely charged globular proteins. Biopolymers 83: $148-158$

10. Lee SH, Heng D, Ng WK, Chan HK, Tan RBH (2011) Nano spray drying: a novel method for preparing protein nanoparticles for protein therapy. Int J Pharm 403:192-200

11. Varca GHC, Ferraz CC, Lopes PS, Beatriz Mathor M, Grasselli M, Lugão AB (2013) Radio-synthesized protein-based nanoparticles for biomedical purposes. Radiat Phys Chem 94:181-185

12. Kim TH, Jiang HH, Youn YS, Park CW, Tak KK, Lee S, Kim H, Jon S, Chen X, Lee KC (2011) Preparation and characterization of water-soluble albumin-bound curcumin nanoparticles with improved antitumor activity. Int J Pharm 403:285-291

13. Ferrado JB, Perez AA, Visentini FF, Islan GA, Castro GR, Santiago LG (2019) Formation and characterization of self-assembled bovine serum albumin nanoparticles as chrysin delivery systems. Colloids Surf B Biointerfaces 173:43-51

14. Fraylich MR, Liu R, Richardson SM, Baird P, Hoyland J, Freemont AJ, Alexander C, Shakesheff K, Cellesi F, Saunders BR (2010) Thermally-triggered gelation of PLGA dispersions: towards an injectable colloidal cell delivery system. J Colloid Interface Sci 344: 61-69

15. Govender T, Stolnik S, Garnett MC, Illum L, Davis SS (1999) PLGA nanoparticles prepared by nanoprecipitation: drug loading and release studies of a water soluble drug. J Control Release 57: $171-185$

16. Varga N, Turcsányi Á, Hornok V, Csapó E (2019) Vitamin E-loaded PLA- and PLGA-based core-shell nanoparticles: synthesis, structure optimization and controlled drug release. Pharmaceutics 11: 357

17. Varga N, Hornok V, Janovák L, Dékány I, Csapó E (2019) The effect of synthesis conditions and tunable hydrophilicity on the drug encapsulation capability of PLA and PLGA nanoparticles. Colloids Surf B Biointerfaces 176:212-218
18. Karnik R, Gu F, Basto P, Cannizzaro C, Dean L, Kyei-Manu W, Langer R, Farokhzad OC (2008) Microfluidic platform for controlled synthesis of polymeric nanoparticles. Nano Lett 8:29062912

19. Capretto L, Carugo D, Cheng W, Hill M, Zhang X (2011) Continuous-flow production of polymeric micelles in microreactors: experimental and computational analysis. J Colloid Interf Sci 357:243-251

20. Jankowski P, Kutaszewicz R, Ogończyk D, Garstecki P (2019) A microfluidic platform for screening and optimization of organic reactions in droplets. J Flow Chem 8:1-12

21. Wojnicki M, Tokarski T, Hessel V, Fitzner K, Luty-Błocho M (2019) Continuous, monodisperse silver nanoparticles synthesis using microdroplets as a reactor. J Flow Chem 9:1-7

22. Vécsei L, Szalárdy L, Fülöp F, Toldi J (2013) Kynurenines in the CNS: recent advances and new questions. Nat Rev Drug Discov 12: 64-82

23. Veres G, Molnár M, Zádori D, Szentirmai M, Szalárdy L, Török R, Fazekas E, Ilisz I, Vécsei L, Klivényi P (2015) Central nervous system-specific alterations in the tryptophan metabolism in the 3nitropropionic acid model of Huntington's disease. Pharmacol Biochem Behav 132:115-124

24. Varga N, Csapó E, Majláth Z, Ilisz I, Krizbai IA, Wilhelm I, Knapp L, Toldi J, Vécsei L, Dékány I (2016) Targeting of the kynurenic acid across the blood-brain barrier by core-shell nanoparticles. Eur J Pharm Sci 86:67-74

25. Varga N, Dékány I, Knapp L, Krizbai I, Majláth Zs, Toldi J, Vécsei L (2020) A process for the delivery of drug molecules through the blood-brain barrier using core-shell nanocomposites, accepted Hungarian patent, P1500356

26. Costa P, Sousa Lobo JM (2001) Modeling and comparison of dissolution profiles. Eur J Pharm Sci13:123-133

27. Dash S, Murthy PN, Nath L, Chowdhury P (2010) Kinetic modeling on drug release from controlled drug delivery systems. Acta Pol Pharm 67:217-223

28. Ritger PL, Peppas NA (1987) A simple equation for description of solute release I. Fickian and non-fickian release from non-swellable devices in the form of slabs, spheres, cylinders or discs. J Control Release 5:23-36

29. Murugeshu A, Astete C, Leonardi C, Morgan T, Sabliov CM (2011) Chitosan/PLGA particles for controlled release of $\alpha$-tocopherol in the GI tract via oral administration. Nanomedicine 6:1513-1528

30. Astete CE, Dolliver D, Whaley M, Khachatryan L, Sabliov CM (2011) Antioxidant poly(lactic-co-glycolic) acid nanoparticles made with $\alpha$-Tocopherol-ascorbic acid surfactant. ACS Nano 5: 9313-9325

31. Zigoneanu IG, Astete CE, Sabliov CM (2008) Nanoparticles with entrapped $\alpha$-tocopherol: synthesis, characterization, and controlled release. Nanotechnology 19:105606

32. Veres P, Kéri M, Bányai I, Lázár I, Domingo C, Kalmár J (2017) Mechanism of drug release from silica-gelatin aerogel-relationship between matrix structure and release kinetics. Colloids Surf B Biointerfaces 152:229-237

33. Kéri M, Forgács A, Papp V, Bányai I, Veres P, Len A, Dudás Z, Fábián I, Kalmár J (2020) Gelatin content governs hydration induced structural changes in silica-gelatin hybrid aerogels - implications in drug delivery. Acta Biomater

Publisher's note Springer Nature remains neutral with regard to jurisdictional claims in published maps and institutional affiliations. 